\title{
A New Electro-Optic Waveguide Architecture and The Unprecedented Devices It Enables
}

\author{
Scott R. Davis, ${ }^{*}$ Scott D. Rommel, George Farca and Michael H. Anderson \\ Vescent Photonics Inc., 4865 E. $41^{\text {st }}$ Ave., Denver CO 80216
}

\begin{abstract}
A new electro-optic waveguide platform, which provides unprecedented electro-optical phase delays $(>1 \mathrm{~mm})$, with very low loss $(<0.5 \mathrm{~dB} / \mathrm{cm})$ and rapid response time (sub millisecond), is presented. This technology, developed by Vescent Photonics, is based upon a unique liquid-crystal waveguide geometry, which exploits the tremendous electro-optic response of liquid crystals while circumventing historic limitations of liquid crystals. The exceedingly large optical phase delays accessible with this technology enable the design and construction of a new class of previously unrealizable photonic devices. Examples include: a 1-D non-mechanical, analog beamsteerer with an $80^{\circ}$ field of regard, a chip-scale widely tunable laser, a chip-scale Fourier transform spectrometer $(<5 \mathrm{~nm}$ resolution demonstrated $)$, widely tunable micro-ring resonators, tunable lenses, ultra-low power ( $<5$ microWatts) optical switches, true optical time delay (up to $10 \mathrm{~ns}$ ), and many more. All of these devices may benefit from established manufacturing technologies and ultimately may be as inexpensive as a calculator display. Furthermore, this new integrated photonic architecture has applications in a wide array of commercial and defense markets including: remote sensing, micro-LADAR, OCT, laser illumination, phased array radar, optical communications, etc. Performance attributes of several example devices are presented.
\end{abstract}

Keywords: liquid crystal waveguides, electro-optic waveguides, photonic devices, tunable micro-ring, non-mechanical beamsteerer, tunable laser

\section{INTRODUCTION}

Numerous applications require active control over light including: robotic-vision, optical computing, telecommunications, holographic data-storage, remote sensing, cold-atom optics, industrial process analysis, and many more. In response to this need a diverse array of technologies have been investigated and developed over the past several decades: micro-electro mechanical systems (MEMs), ${ }^{1}$ photonic crystals, ${ }^{2}$ thermo-optics, ${ }^{3}$ and electro-optic materials such as inorganic crystals and organic poled-polymers. ${ }^{4}$ While tremendous progress has been made, there are still numerous applications, such as beamsteering and large optical phase delay, where bulky and power-consumptive macroscopic opto-mechanical techniques are still the best. This is, at least in part, because typical electro-optic approaches do not realize sufficient control over light $(R$ matrix values of typically $<100 \mathrm{pm} / \mathrm{V})$ to replace traditional opto-mechanics. MEMs techniques are still inherently mechanical, which imposes vibration and inertia design challenges, and developing MEMs architectures that provide macroscopic $(>1 \mathrm{~mm})$ control over optical phase has been challenging.

Vescent's proprietary liquid-crystal(LC) waveguide architecture ${ }^{5-9}$ provides unprecedented voltage control over optical phase $(>1 \mathrm{~mm})$, orders of magnitude more than any other technology, e.g., liquid crystal optical phased arrays ${ }^{10}$ or MEMs. This previously unrealizable level of control makes possible new devices with remarkable performance attributes. To date we have demonstrated: ultra-wide field of view $\left(80^{\circ}\right)$ non-mechanical laser beamsteerers, FTIR spectrometers on a chip with $<5 \mathrm{~nm}$ resolution, chip-scale widely tunable lasers (nearly $40 \mathrm{~nm}$ tunability demonstrated), ultra-low power $(<5 \mu$ Watts) tunable micro-ring filters and Mach-Zehnder switches, and many more. All of these devices may be in small LCD-like packages that can ultimately be as low cost as a calculator display.

\footnotetext{
* Corresponding author. Tel.: 303-296-6766; fax: 303-296-6783.

Email address: davis@vescentphotonics.com 


\section{THE ENABLING INNOVATION}

Over the past several decades one of the most technically and commercially successful approaches for light control has been liquid-crystal (LC) optics. LCs have the world largest electro-optic response ( $\Delta n>0.2$ over 5 volts for a typical LC, which corresponds to $10^{5}-10^{6} \mathrm{pm} / \mathrm{V}$, i.e., several orders of magnitude larger than any other approach), are environmentally stable, amenable to high-volume manufacturing, and inexpensive. ${ }^{11}$ This has enabled the now $>\$ 50$ billion a year display market. A typical "display-like" LC-optic is shown in Figure 1. The light traverses a thin $(<20$ $\mu \mathrm{m}) \mathrm{LC}$ layer that is sandwiched between glass sheets. Transparent electrodes are used to apply an electric field, which, in combination with polarizers, may be used to either block or transmit the light.

While undeniably potent for information displays, this traditional LC-optic has two significant limitations. First, the light must transmit through transparent electrodes, which in turn limits the total optical power that may be controlled. Second, and arguably more significant, the LC layer must be extremely thin. The LC- material is rendered a single-domain crystal via thin alignment layers. The LC-molecules adjacent these alignment layers are highly ordered, which means low scattering loss, and fast response to changes in voltage. If one were to make the LC cell thicker, the bulk LC material would become prohibitively slow and opaque. Therefore, even though the LC material has a tremendous electro-optic effect, the necessarily short interaction length mitigates this effect. In order to circumvent these limitations we have invented and are developing the LC-clad waveguide architecture.

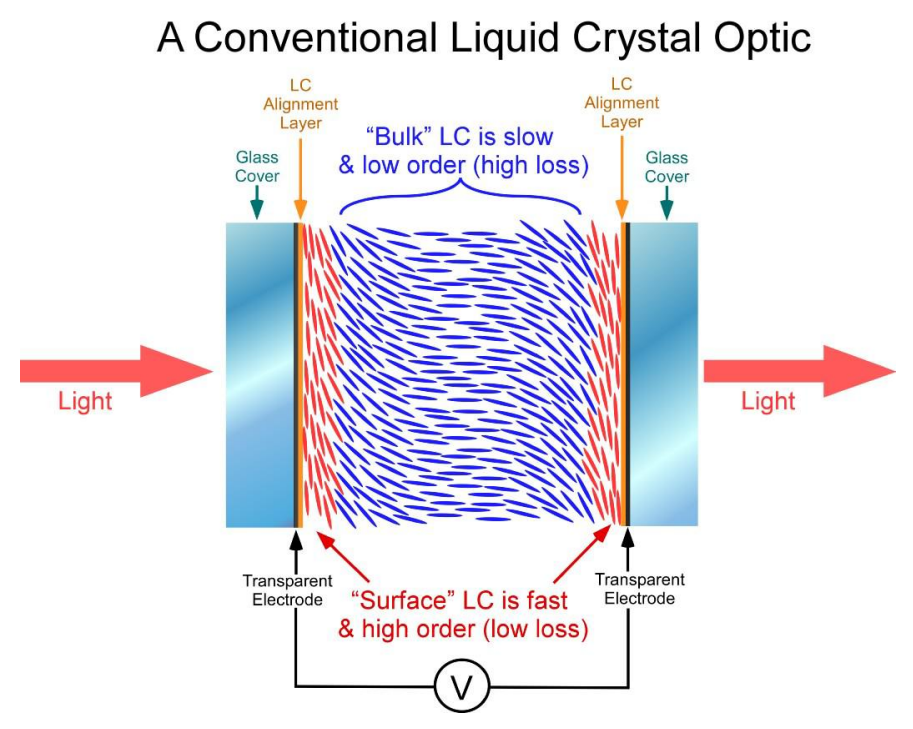

Figure 1: A Typical LC-Optic, such as is used in the ubiquitous LC-Display.

\subsection{Giant control over optical phase: $L C$-waveguides}

Rather than transmit through an LC cell, which by design must be thin (typically $<20 \mu \mathrm{m}$ ), we utilize the LC as an active cladding layer in a waveguide architecture, i.e., the light skims along the surface of an LC layer, as shown in Figure 2. This electro-evanescent architecture circumvents limitations of traditional LC-optics: i) the light never crosses a transparent electrode, ii) the light only interacts with the well-behaved LC-surface layer via the evanescent field, and iii) the interaction length is now decoupled from the LC-layer thickness.

For a given liquid crystal and waveguide structure we have modeled the LC upper cladding and the voltage dependent field profile of the guided light. This is shown in Figure 3. Specifically, our model includes: LC surface energy, pretilt, elastic coefficients of the LC, electrical properties of the LC (dielectric constants), optical properties of the LC (birefringence), electrode spacing, and electrical properties of the waveguide materials. With this information we have numerically solved for the LC upper cladding index profile as a function of voltage, following an established routine outlined by S. T. Wu. ${ }^{11}$ Then, for a given index profile we solve Maxwell's equation for the guided mode and determine the effective waveguide index. The index modulation is the magnitude of the difference between the effective index at zero volts and the effective index and a higher voltage. This model does an excellent job of predicting the experimental results. Furthermore, the model shows that $\Delta n_{\text {eff }}=0.05$ is possible by using highly-birefringent liquid crystals and by keeping the ratio of core thickness to wavelength less than one. While this represents a four-fold decrease in birefringence from the raw liquid crystal, this is more than offset by a possible 10,000-fold increase in the interaction length. 
Example operation of an LC-waveguide optical phase shifter is given in Figure 4. The core of the waveguide is LPCVD deposited $\mathrm{Si}_{3} \mathrm{~N}_{4}(n=2.0)$ and was approximately $500 \mathrm{~nm}$ thick, the sub-cladding is $\mathrm{SiO}_{2}$ and the substrate is a P-doped $\mathrm{Si}$ wafer. This device exhibited more than 1 millimeter of voltage tunability over optical phase. We know of no other technology that can provide similar performance. Furthermore, the LC waveguide switching time is faster than normal liquid crystals by about one order of magnitude. Typical relaxation times for LC waveguides are on the order of 500 $\mu \mathrm{sec}$, though as will be shown this can be further reduced via choice of operational voltages and device design.

\section{Liquid Crystal Clad Waveguides}

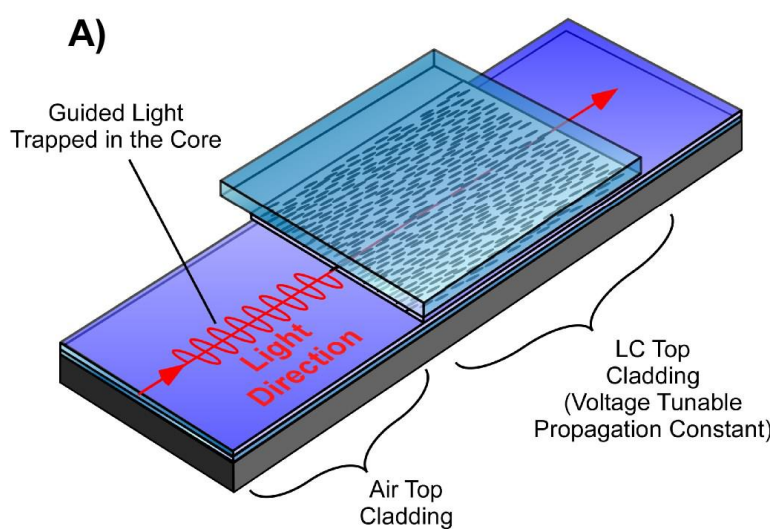

B)

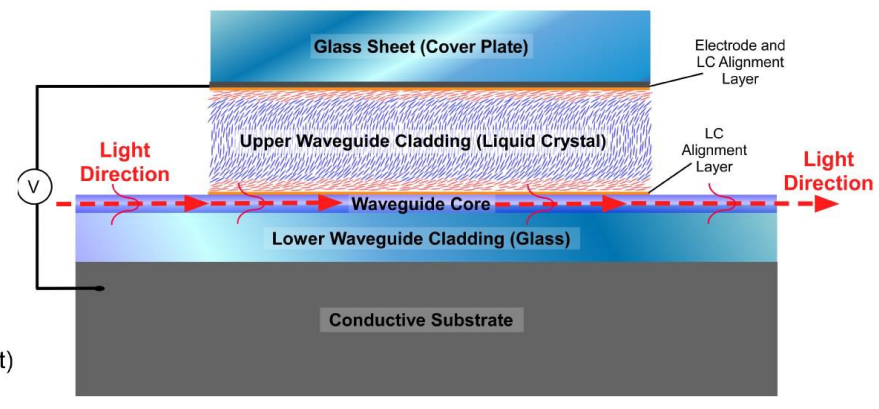

Figure 2: A) The basic geometry of an LC-waveguide. The light is confined to a core and the LC is an electrooptic upper cladding. As the index of refraction of the upper cladding is tuned the "effective index" of the guided mode is also tuned. B) A side view of a liquid crystal waveguide. In a slab waveguide the light is guided in the $x$ dimension, but is free to propagate as Gaussian beams, sheets, or even 1D images in the plane.

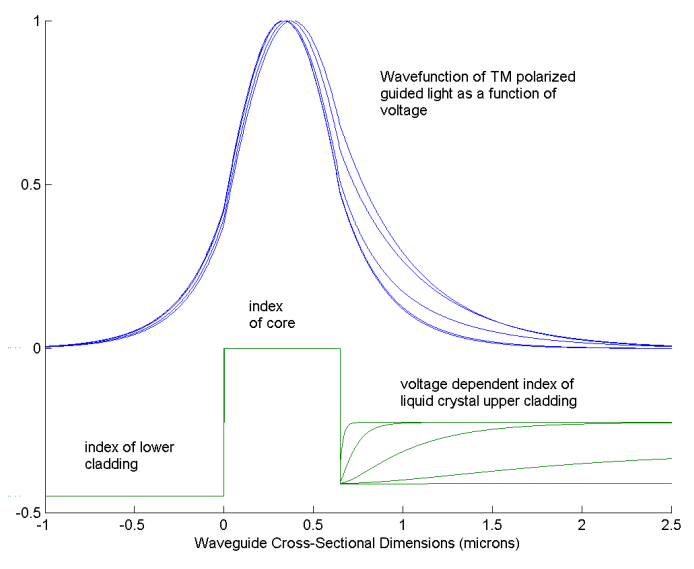

Figure 3: The green lines show the calculated index profile of an LC clad waveguide for different applied voltages. As the voltage is increased the index of the upper cladding also increases. The blue lines show the intensity profile for TM light as a function of voltage. This was obtained by direct solving of Maxwells equations for the waveguide boundary conditions.
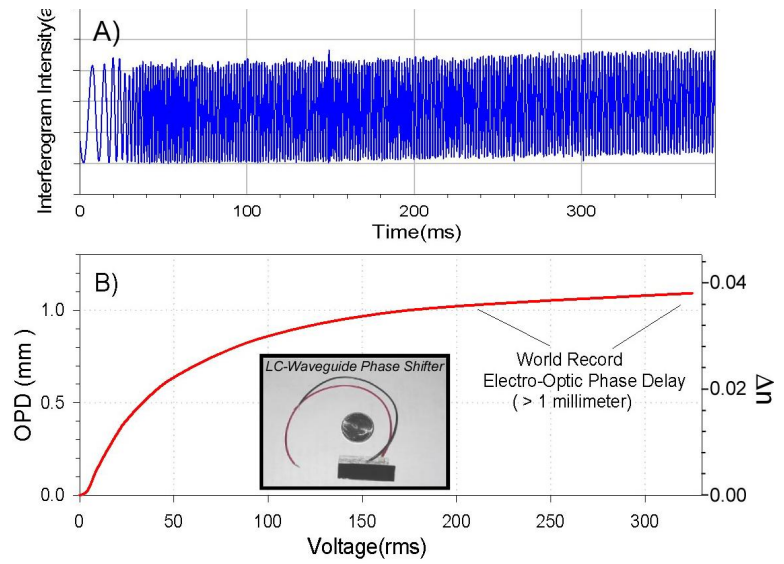

Figure 4: The performance of an LC waveguide filled with a nematic liquid crystal with a birefringence of about $\Delta \mathrm{n} \sim 0.2$. A) The transmission of the $\mathrm{LC}$ waveguide between polarizers. The figure was recorded over a longer sweep time so that individual waves could be observed. B) The tunable optical phase delay versus applied voltage. For this device greater than one millimeter of OPD was achieved. 


\section{NEW PHOTONIC DEVICES}

This extremely large OPD makes possible a whole new class of photonic devices. Figure 5 illustrates a few possibilities. In addition to these LC-waveguides may be used for optical coherence tomography, true optical time delays, tunable lenses, optical switches, and many more. In the rest of this proceedings we provide some examples of new photonic devices that are enabled by LC-waveguides: i) a chip-scale non-mechanical Fourier Transform Spectrometer, ii) nonmechanical wide angle beamsteerers, iii) chip-scale widely tunable lasers, iv) ultra-low power optical switch, and v) voltage tunable micro-ring resonator. These are only some of the devices that we are developing at Vescent and only a small subset of possible LC-waveguide devices.

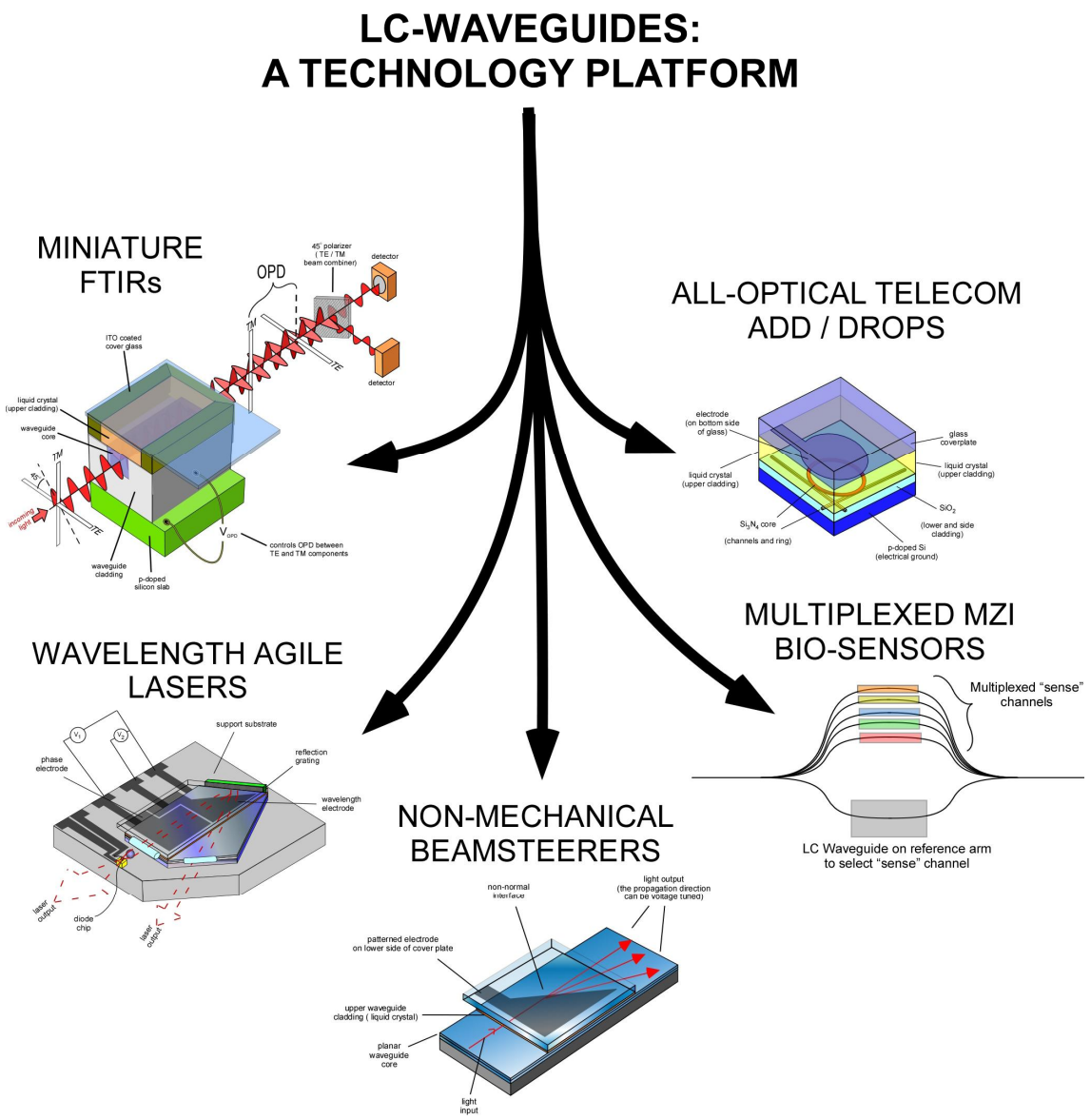

Figure 5. The unprecedented electro-optic phase delays provided by LC-waveguides enable a vast array of new photonic devices. Some examples shown here are: chip-scale polarization based FTIR, telecom tunable optical add/drops (ROADMS), tunable lasers (non-mechanical ECDLs), multiplexed Mach-Zender bio sensors, and a new type of non-mechanical beamsteerers. We have demonstrated prototypes for many of these applications.

\subsection{Chip-Scale Fourier Transform Spectrometer}

A device that demonstrates the utility of giant electro-optic control is a completely non-mechanical Fourier transform spectrometer (FTS). When the LC-waveguide is placed between polarizers the transmitted intensity undergoes extrema as the voltage across the LC layer is tuned. Different wavelengths experience different numbers of extrema and this can be used to analyze the wavelength content of the light. Figure 6 illustrates a non-mechanical FTS prototype developed for NASA. 


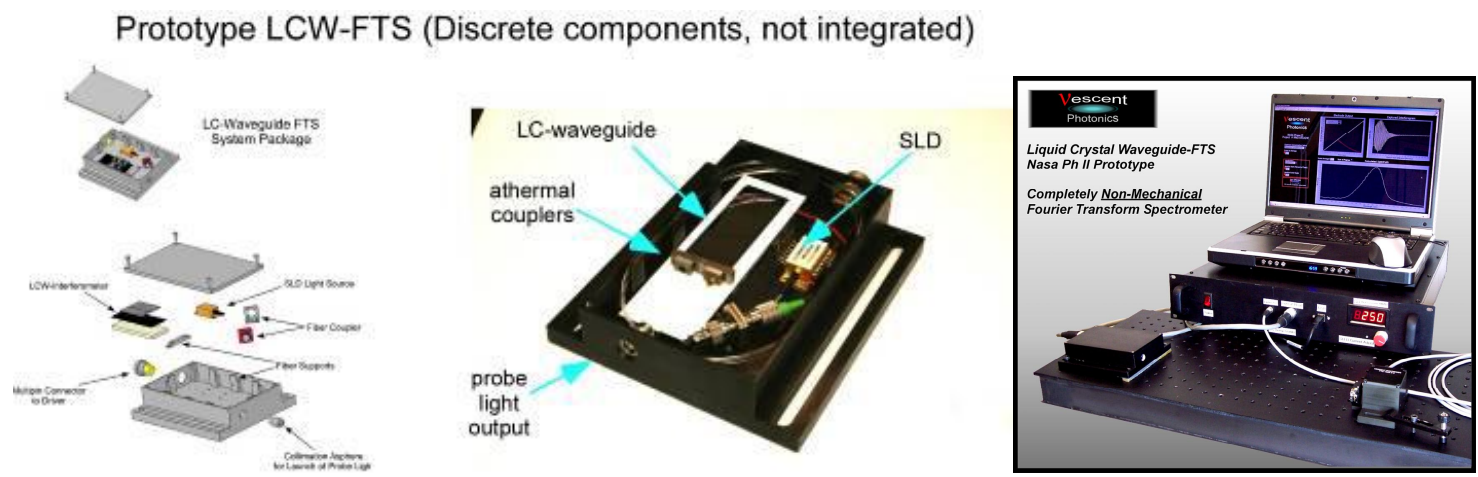

Figure 6: The prototype LC-Waveguide FTS system for operation in the near-IR. This current prototype utilizes a bulky electronics driver and laptop for control and data analysis. This may be replaced with mircro-electronics.

Example performance of the prototype device is shown in Figure 7. The left figure depicts LC-waveguide obtained interferograms of narrow-band light sources. The bottom interferogram is for a single frequency laser. The top interferogram is for two lasers. The beat pattern of the two lasers is clearly visible in the interferogram. The insets show the spectrum of the light sources. The figure on the right shows interferograms and FFT obtained spectra of a broadband SLD with some broadband spectral absorption features. In these experiments both filter and acetylene absorption spectra were recorded. For these devices the spectral resolution was 3-5 nm and the spectral coverage was in the near-IR range from 1450-1700 nm. New designs improve the spectral resolution to $<0.5 \mathrm{~nm}$.

\section{Example Performance of the LCW-FTS Prototype}

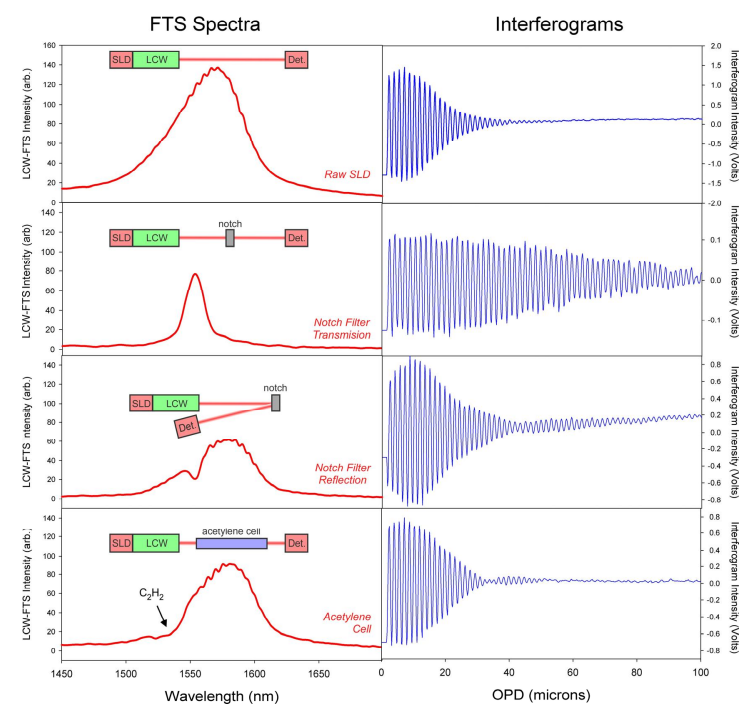

Figure 7. The figure on the left shows an LC waveguide Fourier transform interferogram. The upper trace shows the interferogram (truncated for clarity) of a multimode diode laser. The lower trace shows the interferogram of a pure wavelength source. The "beat-note" for the multimode laser is clearly visible in the top trace. The figure on the right shows the interferogram from a broadband SLD along with the FFT spectra for a variety of absorption features.

\subsection{Electro-Optic Laser Scanners}

The challenge of non-mechanical beam control is a long-standing one, ${ }^{10,12-18}$ and has been the subject of extensive past efforts (e.g., The Steered Agile Beam or STAB project ${ }^{19}$ funded by DARPA in 2000). A diverse array of technical approaches have been directed toward this problem including: i) planar electro-optic prisms constructed from KTP, ${ }^{20}$ Lithium Niobate, ${ }^{20}$ ferroelectric domain $\mathrm{LiTaO}_{3},{ }^{17,21}$ and electro-optic polymers,${ }^{22}$, ii) thermo-optic planar prisms, ${ }^{23}$ iii) diffractive liquid crystal phased arrays ${ }^{10,18}$, and iv) diffractive acousto-optic techniques ${ }^{24}$. Each of these approaches has 
advantages and drawbacks. Electro-optic crystals are very fast but have extremely small electro-optic coefficients, which means very small steering angles and kilovolts to operate. Furthermore, EO crystals are quite expensive. Liquidcrystal optical-phased array beamsteerers tend to be slow, provide non-continuous diffractive steering, and have a very limited steering range because thick LC layers are problematic. Acousto-optic beamsteerers have a larger steering range but are also diffractive, require very large power supplies and expensive crystals.

LC-waveguides provide an entirely new approach to electro-optic laser-beamscanning. ${ }^{5}$ The basic concept is shown in Figure 8 wherein a lithographically patterned electrode provides a 1-D prism whose refractive index can be tuned relative to the surrounding area, providing truly analog steering, i.e., it is not diffractive. This lithographically patterned electrode is similar to, albeit much simpler than, the patterned electrodes required for traditional liquid crystal displays. In principle, the pointing precision is only limited by the noise on the control voltage.

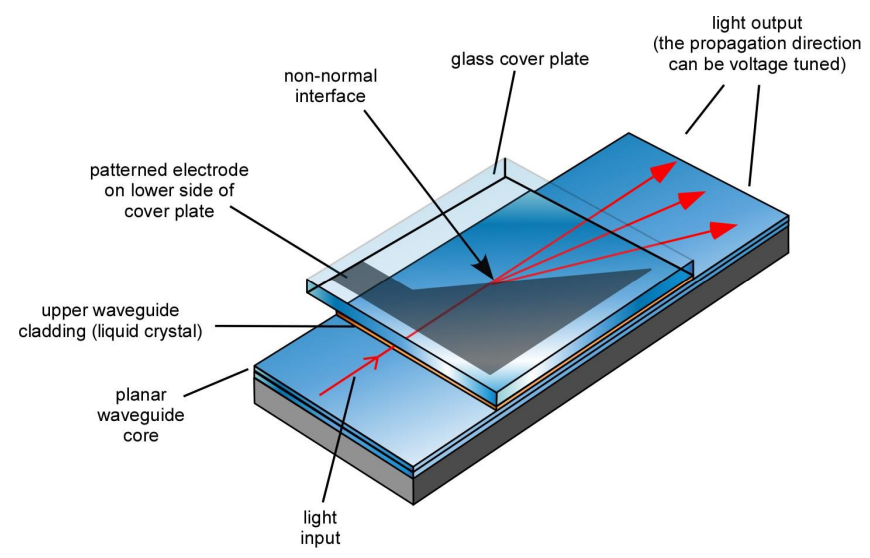

Figure 8: A 1-D LC-waveguide beamsteerer. A single control voltage is applied to a prism-shaped electrode having a non-normal interface to the beam propagation direction. As voltage is applied, the index under the patterned electrode is changed relative to the surrounding area and the beam is steered via Snell's law refraction.

We have designed and built several prototype LC-waveguide beamsteerers. In order to realize a larger deflection angle each steer electrode is patterned to have multiple interfaces in series. In this way the amount of deflection is accumulated along the length of the waveguide. The vertical dimensions of the later electrodes may be expanded to
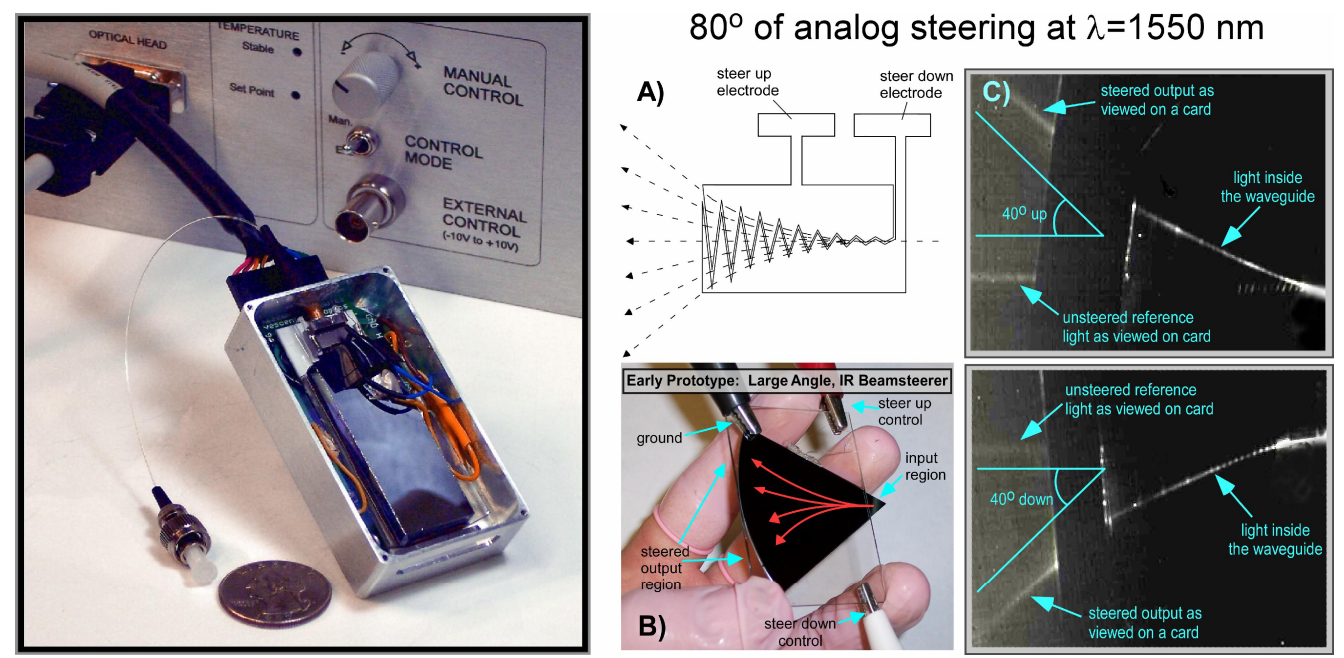

Figure 9 (Left) A picture of one our commercial prototypes for compact, non-mechanical wide angle

beamsteerering. (Right) A: The lithographically patterned electrode for controlling beam deflection. B: A picture of a large-angle electro-optic beamsteerer. The red lines are drawn to help guide the eye. This device was made on $\mathrm{Si}$, but it could have been made on glass. C: Images recorded with an InGaAs CCD camera of the prototype beamsteerer in action. The device produced $80^{\circ}\left( \pm 40^{\circ}\right.$ of non-mechanical beamsteering, with only two control voltages). 
prevent optical clipping of the steered beam, as is discussed in ref ${ }^{17}$. An example of a multiple interface electrode pattern is shown in Figure 9A. A picture of a prototype waveguide device is shown in Figure 9B, and Figure 9C shows the performance as viewed with a high-gain InGaAs CCD camera. A small amount of non-steered light was deliberately allowed onto the exit card, so an accurate beamsteering angle could be measured. This device exhibited a total analog steer range of over $80^{\circ}$ for a $1 \mathrm{~mm}$ input aperture, with only two control electrodes. To our knowledge this is the largest electro-optic steering ever realized for a two-electrode device. Performance examples of LC-waveguide beamsteerer devices are shown in several short videos online (http://www.vescentphotonics.com/SEEOR.html).

\subsection{Chip-Scale Widely Tunable Lasers}

LC-waveguides may be used to construct tunable filters by placing beamsteering elements upstream from edge-bonded reflection gratings, or by placing LC over waveguides with Bragg gratings or ring resonators. An application of a tunable filter is for a tunable diode laser. We have exploited the giant electro-optic effect to replace the mechanical components in an external cavity diode laser in Littrow configuration. One electrode controls the tuning wavelength and the other controls the optical path length of the cavity enabling mode-hop-free tuning over very large ranges. The device is completely electronic, robust, and low cost. The upper left picture in Figure 10 shows the design of a tunable diode laser based on a tunable Littrow grating filter. The lower left of Figure 10 shows an example of a prototype LCwaveguide laser. Tuning performance is shown in the right of Figure 10.
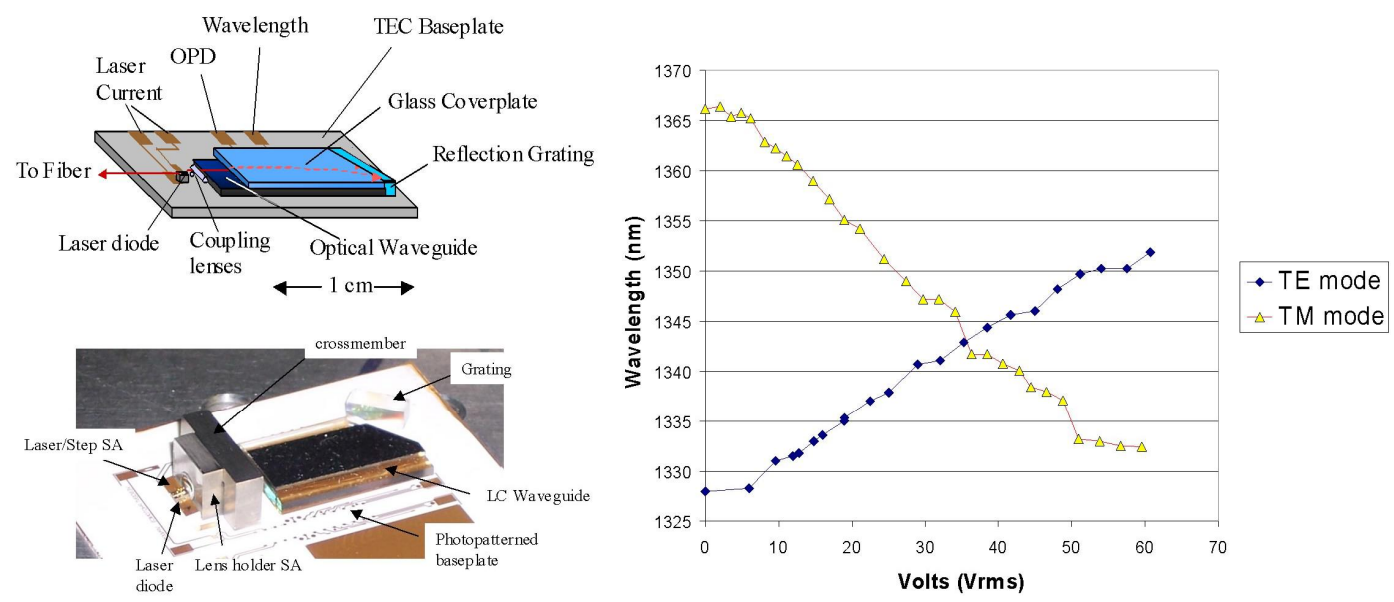

Figure 10. An LC waveguide tunable diode laser and some recent performance data. The upper left hand picture shows a drawing of the basic structure. A typical external cavity laser architecture is integrated onto an LCwaveguide. A picture of a micro-assembly of a laser is shown in the lower left hand corner. The plot on the right shows experimental wavelength tuning with voltage. The jumps or discontinuities in the tuning are due to laser mode-hops, which can be mitigated with new laser designs.

\subsection{Ultra-Low Power Optical Switch}

LC-waveguide may also be used to provide a low power alternative to thermo-optic waveguide devices. As an example we have built prototype LC-waveguide $2 \times 2$ Mach Zehnder Interferometer (MZI) switches. A microscope picture of the device and experimental operation is shown in Figure 11. In this example light from a single frequency laser was coupled into one of the entrance ports. While voltage to the LC-waveguide was ramped the intensity at an exit port was monitored. As can be seen from the plot the index modulation was sufficient to scan through several minima and maxima, i.e., it was enough to switch the device several times. Further device performance is shown in Figure 12 and Table 1. This device, developed in collaboration with LioniX photonics, utilized a Triplex ${ }^{25}$ LPCVD channel waveguide structure, with an active LC cladding. These devices provide substantial improvements over thermo-optic designs: i) larger index modulation which permits a more compact switch, ii) faster switching time by more than an order of magnitude over thermo-optics, and iii) a factor of 10,000 less electrical power consumption. The electrical driving requirements for $\mathrm{LC}$-waveguide devices are equivalent to driving a small capacitor, typically a few nanofarads. 
Low-Cost Voltage Tunable Optical Switch

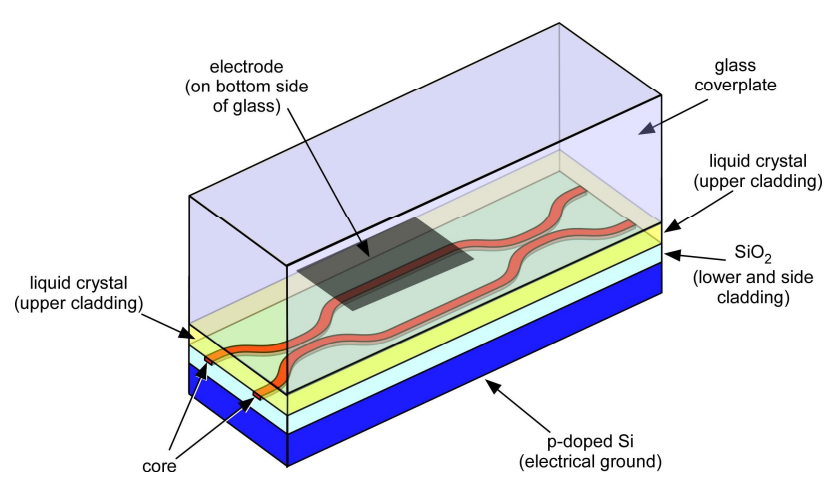

Example Performance of LC-Clad

Mach-Zehnder 2x2 Switch

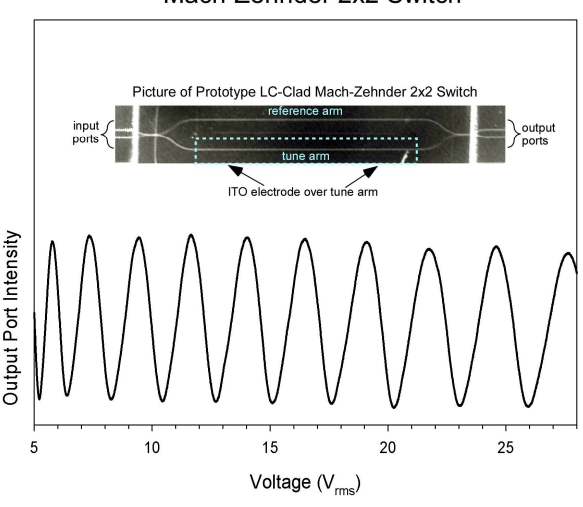

Figure 11: Left: Close-up view of a single voltage tunable MZI switch. This is analogous to a single pixel on an LC display. The planar waveguide sub-structure is constructed from well established silica on silicon technology. The top cladding contains liquid crystal, which may be voltage tuned, thereby tuning the optical phase delay of the MZI tune arm. Right: Example performance and picture (inset) of a voltage tunable $2 \times 2$ MZI optical switch. The separation between the reference and tune arm was $500 \mu \mathrm{m}$. Light was fiber coupled into the top left port and the bottom right port was monitored as a function of voltage. The light wavelength was $1550 \mathrm{~nm}$.
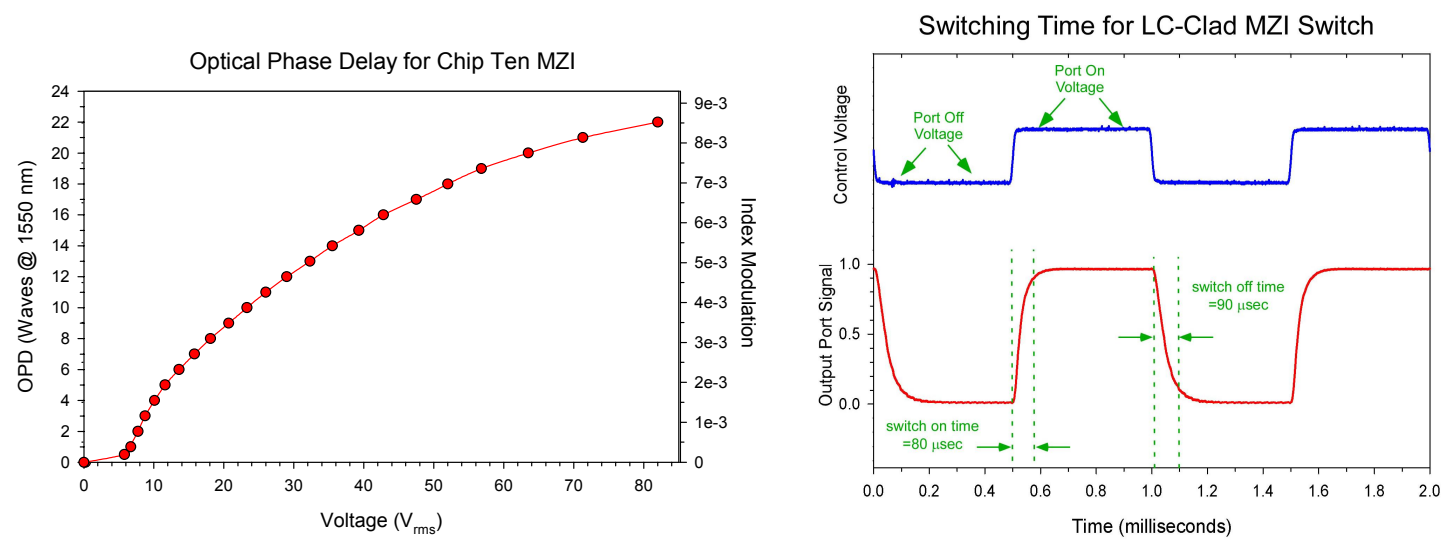

Figure 12: Shown on the left is the voltage tunable optical phase delay and index modulation realized with this LC-waveguide architecture. Shown on the right are switching times. The control voltage is a DC balanced square wave, whose amplitude is altered. For this switching data the amplitude was altered between approximately 100 and 80 volts. Voltage driving at lower values will yield a slower response $(\approx 400 \mu \mathrm{sec})$, but still faster than typical thermo-optics (e.g. 5 milliseconds).

Table 1: Measured performance of LC-Waveguide 2×2 MZI optical switch

\begin{tabular}{c|c} 
Property & Value \\
\hline \hline Switching Speed & $\begin{array}{c}<100 \mu \text { sec for } 100 \mathrm{~V}_{\text {rms }} \text { operation } \\
<400 \mu \mathrm{sec} \text { for } 15 \mathrm{~V}_{\text {rms }} \text { operation }\end{array}$ \\
\hline Power Consumption & $\begin{array}{c}<30 \mu \mathrm{Watts} \text { measured } \\
<3 \mu \mathrm{Watts} \text { expected }\end{array}$ \\
\hline Index Modulation & $\Delta n=7 \times 10^{-3}$ by $50 \mathrm{~V}_{\mathrm{rms}}$ \\
\hline LC-Waveguide Loss & $<0.3 \mathrm{~dB} / \mathrm{cm}$ (upper limit) \\
\hline \hline
\end{tabular}




\subsection{Voltage Tunable Micro-Ring Resonator}

Finally, another type of thermo-optic device that may be rendered electro-optic with the LC-waveguide architecture is the tunable micro-ring resonator. The left side of Figure 13 shows the basic architecture of the voltage tunable LCwaveguide micro-ring. The right of Figure 13 shows a microscope picture of the micro-ring device along with the resonance spectrum of the micro-ring. The channel structures were constructed using the triplex waveguide design (this was on the same wafer as the MZI switches). The input and output channels were three microns wide and the channel in the ring was 15 microns wide and a lateral coupling scheme was used. While the ring channel width was not single mode
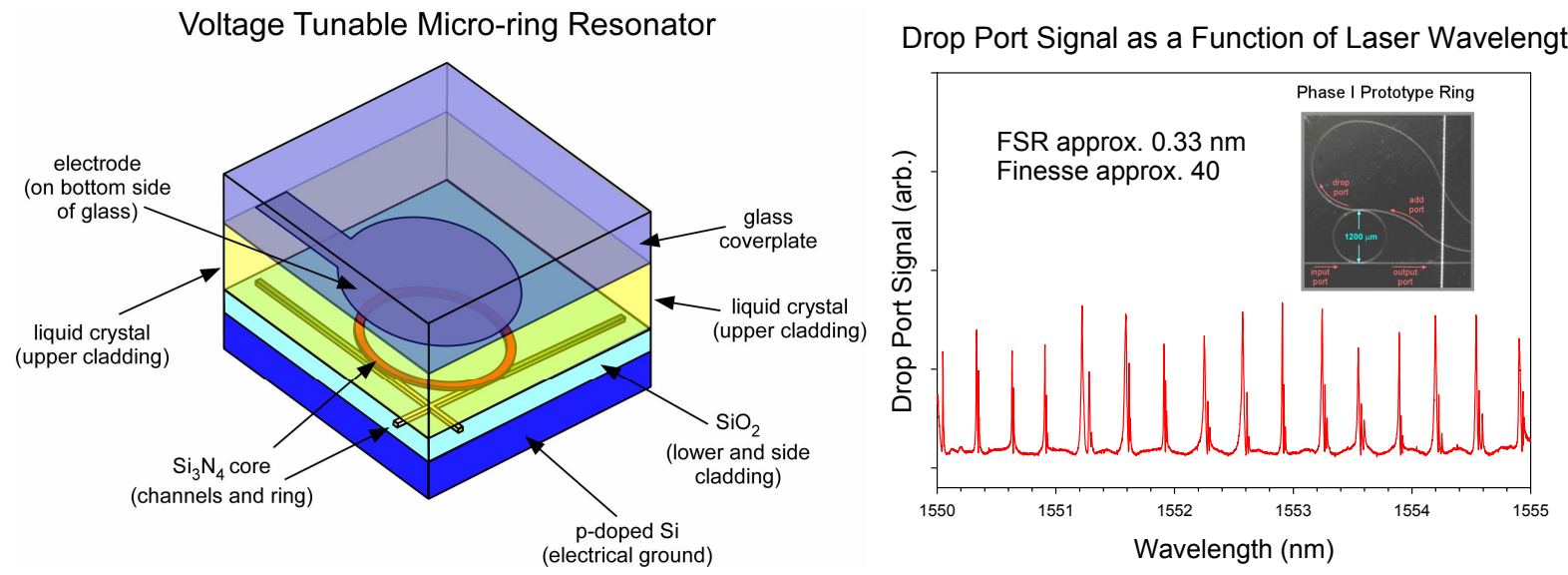

Fig 13. Left: Close-up view of a single voltage tunable micro-ring resonator. This is analogous to a single pixel on an LC display. The planar waveguide sub-structure is constructed from silica on silicon technology. The top cladding contains liquid crystal, which may be voltage tuned, thereby tuning the effective index of the waveguide ring. When tuned to resonance for a given wavelength of light within an input channel, the light can be transferred from one straight channel, through the ring, to the other straight channel. Right: A prototype LC-waveguide micro-ring demonstration. The inset shows a microscope image of the device, and the plot shows the intensity of the drop-port signal as a function of input port wavelength (we used a single mode tunable laser). In this case the voltage to the $\mathrm{LC}$ was held at zero. The ring design only provided a finesse of 40, and the ring was multi-modal. Nevertheless, we were able to use this device to demonstrate voltage tuning of the LC-waveguide ring architecture.

it is our experience that improper coupling between the input channel and the rings is the most common failure mode for first designs. The large channel width in the ring and the large ring diameter were specifically chosen to help mitigate this risk. The spectral response of the ring was measured both with and without LC in the top cladding with no noticeable change in the finesse, indicating that LC scattering losses were minimal.

Voltage tunability of the device and switching speed are shown in Figures 14 and 15 . With the wavelength held fixed at $1550 \mathrm{~nm}$, the intensity of the drop port was monitored as a function of voltage. The results are plotted in Figure 14. As can be seen the device tuned over nine resonances $(>3 \mathrm{~nm})$, with extremely minimal power consumption $(<50 \mu \mathrm{Watts})$. The reason that the peak widths appear to broaden at higher voltages is due to the non-linear index modulation with voltage. Measurement of finesse as a function of voltage showed no discernable change. Also, this finesse was the same with $\mathrm{SiO}_{2}$ as a top cladding, indicating that $\mathrm{LC}$ induced losses are not yet contributing. Other performance attributes are shown in Figure 15. 


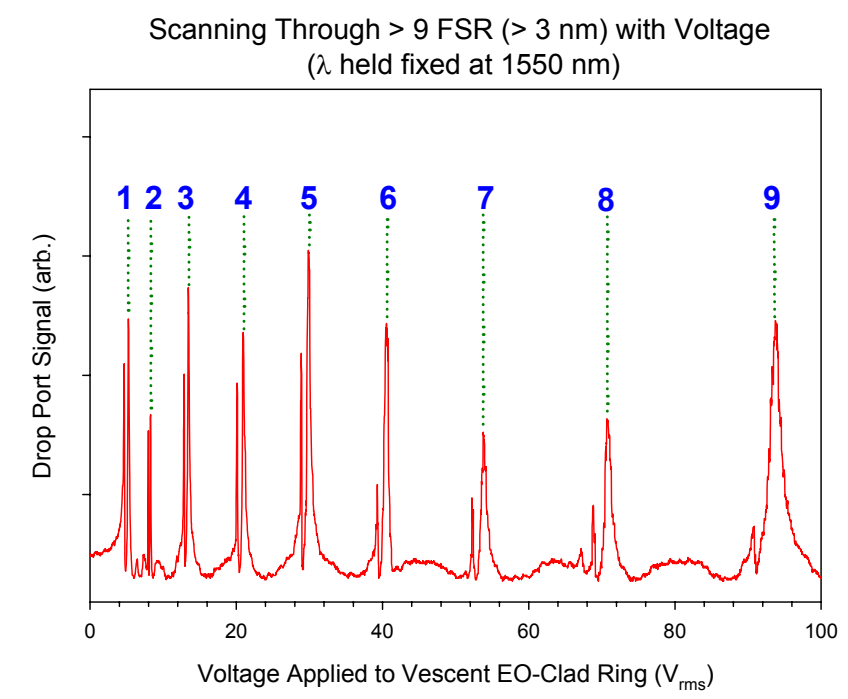

Fig. 14: The wavelength is held fixed at $1550 \mathrm{~nm}$. The voltage on the LC-clad ring is swept from 0 to 100 Volts. As can be seen, $>9$ FSR $(>3 \mathrm{~nm})$ are tuned across. This is for a $600 \mu \mathrm{m}$ radius ring.
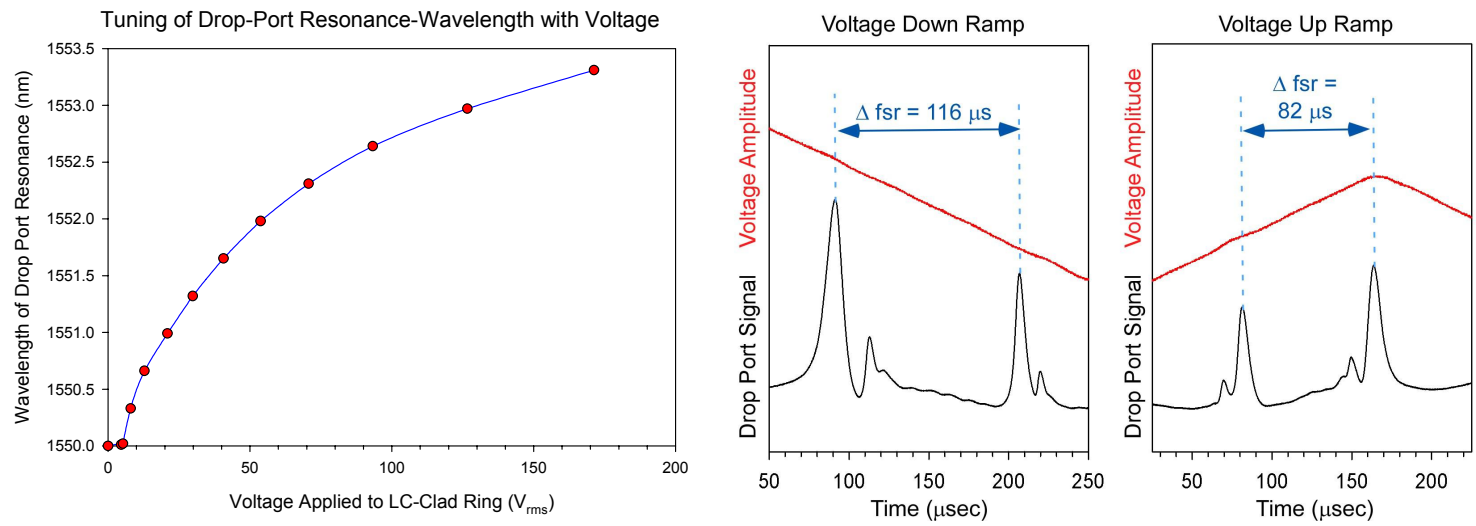

Fig. 15: For the same transmission mode, the resonance wavelength vs. applied voltage is plotted on the left. This is for the $600 \mu \mathrm{m}$ ring. This level of tuning agrees well (within 5\%) with theoretical predictions. The temporal response is shown on the right, both for a voltage increase and decrease (typically with LC's the voltage decrease is the slower response). The tuning times are comparable to those measured for the MZI switch, which is also expected. The different resonance heights, multi-modal resonance, and varying baseline are a property of the ring-resonator foundry run, and not induced by the LC.

\section{CONCLUSION AND FUTURE WORK}

The LC-waveguide architecture is a new electro-optic approach that provides unprecedented voltage control over optical phase $(>1 \mathrm{~mm})$. This previously unrealizable level of control makes possible new devices with remarkable performance attributes. To date we have demonstrated: FTIR spectrometers on a chip with $<5 \mathrm{~nm}$ resolution, widely tunable nonmechanical beamsteerers, chip-scale widely tunable lasers (nearly $40 \mathrm{~nm}$ tunability demonstrated), ultra-low power tunable micro-ring filters and Mach-Zehnder switches. All of these devices may ultimately be in small LCD-like packages that can ultimately be as low cost as a calculator display. At Vescent we are continuing to develop these and other LC-waveguide devices. Future efforts include: full 2-D EO beamsteerers, active polarization cancellation in tunable MZI switches, improved tunable lasers, true time delay devices, and more. 


\section{ACKNOWLEDGMENTS}

This work has been supported by the Air Force Office of Scientific Research, the National Science Foundation, the National Institute of Standards and Technology, and the Environmental Protection Agency. The authors also wish to acknowledge the Colorado Advanced Photonics Technology Center (http://www.captcenter.org), Charles Lee at AFOSR, and Don Snyder at AFRL for their help with the beamsteerer development, and David Walba and Eva Korblova at the University of Colorado Boulder for help with custom liquid crystal chemistries. The authors also wish to acknowledge Rene Heideman and Arne Leinse at LioniX Photonics for their invaluable help with the micro-ring and MZI switch design and fabrication.

\section{REFERENCES}

[1] Wu, M., Solgaard, O., and Ford, J. E. "Optical MEMS for Lightwave Communication," Journal of Lightwave Technology 24, 4433-4454 (2006)

[2] Summers, C. J., Neff, C. W., and Park, W., "Active Photonic Crystal Nano-Architectures," Journal of Nonlinear Optical Physics and Materials 12, 587-597 (2003)

[3] Brainard, R., Fondeur, B. and Dougherty, D. J., "Advances in Planar Lightwave Circuits for Wavelength Routing Applications," in OSA Integrated Optics Conference, (2006)

[4] Jin, D., Dinu, R., Parker, T. S., Barklund, A. Bintz, L., Chen, B., Flaherty, C., Guan, H. W., Huang, D., Kressbach, J., Londergan, T., Mino, T. D., Todorova, G. and Yang, S. "Material development and processing for electro-optic device systems," Proceedings of SPIE, Organic Photonic Materials and Devices, 4991, 610-620 (2003)

[5] Anderson, M., Davis, S. R. and Rommel, S., "Liquid Crystal Waveguide having Refractive Shapes for Dynamically Controlling Light," United State Patent Application, Vescent Photonics, Inc., US (2004)

[6] Anderson, M., Davis, S. and Rommel, S., "Tunable laser having liquid crystal waveguide," United State Patent Application, Vescent Photonics, Inc., US (2005)

[7] Anderson, M., Davis, S. and Rommel, S.,, "Liquid Crystal Waveguide for Dynamically Controlling Polarized Light," United State Patent Application,Vescent Photonics, Inc., US (2005)

[8] Anderson, M., Davis, S. and Rommel, S., "Liquid Crystal Waveguide Having Electric Field Oriented for Controlling Light United State Patent Application,Vescent Photonics, Inc., US (2006)

[9] Anderson, M., Davis, S. and Rommel, S., "Liquid Crystal Waveguide Having Two or More Control Voltages for Controlling Polarized Light," United State Patent Application,Vescent Photonics, Inc., US (2006)

[10] McManamon, P. "An overview of optical phased array technology and status," Liquid Crystals: Optics and Applications 5947, (2005)

[11] Khoo, I. C. and Wu, S. T., [Optics and nonlinear optics of liquid crystals], Hong Kong, World Scientific Publishing, (1993)

[12] Chiu, Y., Zou, J., Stancil, D. D., and Schlesinger, T. E., "Shape-Optimized electrooptic beam scanners: Analysis, design, and simulation," Journal of Lightwave Technology, 17, 108 (1999)

[13] Finlan, M. K., Flood, K. M., Gerard, M. E. ,and Lehman, J. G., "Nonmechanical beam steering using spatial multiplexing," Proceedings SPIE, 3131, 156 (1997)

[14] Gahagan, K. T., Scrymgeour, D. A., Casson, J. L., Gopalan, V., and Robinson, J. M., "Integrated high-power electro-optic lens and large-angle deflector," Applied Optics, 40, 5638 (2001)

[15] Khan, S. A., and Riza, N. A., "Demonstration of 3-dimensional wide angle laser beam scanner using liquid crystals," Optics Express, 12, 868 (2004)

[16] Revelli, J. F., "High-resolution electrooptic surface prism waveguide deflector: an analysis," Applied Optics 19, $389(1980)$ 
[17] Scrymgeour, D. A., Barad, Y., Gopalan, V., Gahagan, K. T., Jia, Q., Mitchell, T. E. and Robinson, J. M., "Large-angle electro-optic laser scanner on $\mathrm{LiTaO} 3$ fabricated by in situ monitoring of ferroelectric-domain micropatterning," Appl. Opt., 40, 6236 (2001)

[18] Stockley, J. E., Serati, S. A., Sharp, G. A., Wang, P., Walsh, K. F., and Johnson, K. M. "Broadband beam steering," Proceedings of SPIE, 3131, 111 (1997)

[19] http://www.darpa.mil/mto/stab/.

[20] Chiu, Y., Burton, R. S., Stancil, D. D., and Schlesinger, T. E. "Design and Simulation of Waveguide Electrooptic Beam Deflectors," Journal of Lightwave Technology, 13, 2049 (1995)

[21] Scrymgeour, D. A., Tian, L., Gopalan, V., Chauvin, D. and Schepler, K. L. "Phased-array electro-optic steering of large aperture laser beams using ferroelectrics," Applied Physics Letters 86, 211113 (2005)

[22] Sun, L., Kim, J. H., Jang, J. H., An, D., Lu, X., Zhou, Q., Taboada, J. M., Chen, R. T., Maki, J. J., Tang, S., Zhang, H., Steier, W. H., Zhang, C., and Dalton, L., "Polymeric waveguide prism-based electro-optic beam deflector," Optical Engineering, 40, 1217-1222 (2001)

[23] Kim, J. H., Sun, L., Jang, C. H., Choi, C. C., and Chen, R. T., "Polymer-based thermo-optic waveguide beam deflector with novel dual folded-thin-strip heating electrodes," Optical Engineering, 42, 620-624 (2003)

[24] Yariv, A., [Quantum Electronics], John Wiley \& Sons, New York, (1989)

[25] Heideman, R. G., Melloni, A., Hoekman, M., Borreman, A., Leinse, A. and Morichetti, F., "Low loss, high contrast optical waveguides based on CMOS compatible LPCVD processing: technology and experimental results.," LEOS Benelux, (2006) 